\title{
Testbed of OBS/GMPLS interworking
}

\author{
(Invited Paper)
}

\author{
Xaiobin Hong, Hongxiang Guo, Jian Wu, Yawei \\ Yin, Lei Liu, Yong Zuo, Kun Xu, Jintong Lin \\ Key Laboratory of Information Photonics and Optical \\ Communications, Ministry of Education \\ Beijing University of Posts and Telecommunications \\ Beijing, China \\ xbhong@bupt.edu.cn
}

\author{
Takehiro Tsuritani \\ KDDI R\&D Laboratories Inc. \\ 2-1-15 Ohara, Fujimino-shi \\ Saitama 356-8502, Japan
}

\begin{abstract}
A testbed of OBS/GMPLS interworking network is established with the supporting of the BUPT-KDDI cooperation project. A dedicated GMPLS border controller with necessary GMPLS extensions and some OBS extensions are introduced in this network to achieve a dynamic, efficient and transparent inter-domain Burst Header Packet (BHP) and Data Burst (DB) transmissions over the GMPLS-controlled Optical Circuit Switching OCS network. The dynamic restoration in the OBS domain and the GMPLS protection/restoration in the OCS network are implemented in this testbed to demonstrate streaming media transmission in the multilayer network with different QoS requirements.
\end{abstract}

Keywords-OBS;GMPLS;

\section{INTRODUCTION}

The remarkable growth of Internet traffic has resulted in demands for larger transmission capacity, multi-granularities transmission and higher switching/processing speed in the network. In order to enable the existing Wavelength switched optical network (WSON) to support both wavelength and subwavelength granularities, Beijing University of Posts \& Telecommunication and KDDI Corporation started a joint project to investigate an overlay-based [1] multi-layer network architecture for interworking the generalized multiprotocol label switching (GMPLS) controlled WSON network with optical burst switching (OBS) networks.

A dedicated GMPLS border controller with necessary GMPLS extensions, including group label switching path (LSP) provisioning, node capability advertisement, and wavelength label [2] as well as wavelength availability advertisement, is introduced in this multi-layer network to enable a simple but flexible interworking operation. The OBS extensions such as virtual link setup latency advertisement and dynamic offset time calculation are introduced in OBS border nodes in order to achieve dynamic, efficient and transparent inter-domain Burst Header Packet (BHP) and Data Burst (DB) transmissions over the GMPLS-controlled OCS network.

This project also investigated the survivability schemes in the network. The dynamic restoration schemes are proposed by introducing burst retransmission scheme in the OBS domain.
Moreover, the interworking between the GMPLS protection/restoration mechanism (i.e. $1+1$, preplan rerouting and full rerouting) and the OBS retransmission scheme is also implemented and evaluated.

In this paper, the multimedia transmission on a dynamically protected transparent OBS/GMPLS network testbed is firstly demonstrated to show the traffic transmission with different QoS requirements. The impacts of different survivability schemes on the streaming media service and the dynamic protection of multiple QoS level of services are experimentally studied.

\section{SETUP OF OBS/GMPLS NETWORK}

Currently, most of the existing transparent optical networks are based on WSON, or wavelength-routed technology. However, the typical long-lived lightpath in WSON networks may suffer low resource utilization if the ever-increasing, short-lived bursty Internet traffic is directly transmitted over it. On the other hand, Optical Burst Switching (OBS) has demonstrated its capability of accommodating bursty traffic into the optical layer in an effective manner [3]. Therefore, it is reasonable to consider deploying the OBS network at the edge of the core network, and then investigate the integration or interworking between the OBS edge network and the WSON core network. The overlay model based OBS/GMPLS interworking network architecture [4] provides a solution for this kind of transparent and efficient optical multilayer network. As shown in Fig. 1, the overlay model is suitable for the interworking architecture considering that a specific GMPLS border controller (GBC) is deployed at the GMPLS edge to interwork with the OBS border node $(\mathrm{BN})$, and no inter-layer routing/signaling between GMPLS and OBS is required. The automatic inter-domain neighbor discovery among OBS client networks over a GMPLS network improves the scalability of multilayer network; the dynamic virtual link (i.e. Group LSP) establishment depending on the traffic volume provides an efficient usage of the bandwidth resources, and the end-to-end transparent BHP/DB transmission over the group LSP simplifies the migration of the interworking architecture [4]. 


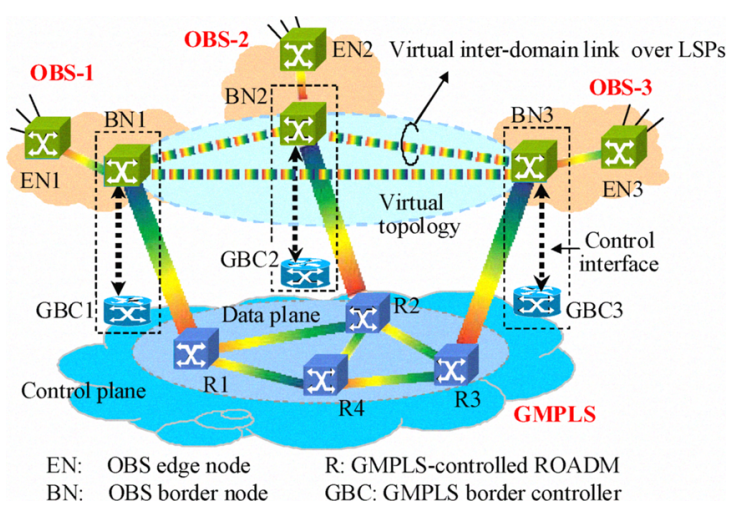

Figure 1 Overlay model for OBS/GMPLS multilayer network.

In order to support the protection and restoration in multilayer network, additional OBS extensions should be introduced [5]. As shown in Fig. 2(a), three novel traffic engineering (TE) link attributes: link setup latency (LSL), link protection type (p-type) and link status, in which LSL represents the setup delay of establishing a DB LSP for the data transportation through the GMPLS network, while the link status can be "inactive", "working", or "protecting" to indicate whether a link is out of service, in service along the working

\begin{tabular}{|c|c|}
\hline Type(=4) & Length \\
\hline Sub-type (=1) & $\begin{array}{c}\text { Length } \\
\text { none / 1P1 / } \\
\text { preplan / reroute }\end{array}$ \\
\hline Protection Type - & Length \\
\hline Sub-type (=2) & Working / Protecting \\
\hline Link Status - & Length \\
\hline Sub-type (=3) & Label $1 /$ Label 2 \\
\hline Wavelenghth type & Length \\
\hline Sub-type (=4) & Time \\
\hline Setup Latency -
\end{tabular}

(a)

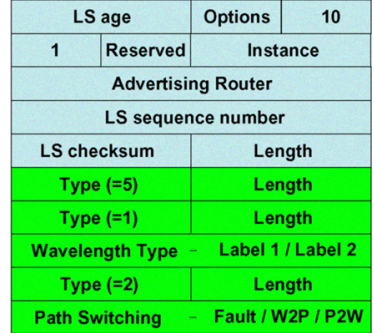

(b)
Figure 2 OBS extensions: (a) TE link attributes of setup latency, protection type and link status, (b) fault notification message.

path, or in service along the protecting path. For the in service "working" or "protecting" link, the LSL equals to 0 . Accordingly, the BHP/DB pair to be scheduled to this link can be assigned with an offset time of basic value at ingress OBS edge node. Note that this basic value is the sum of processing delay (PD) experienced at all transit nodes along the path. On the contrary, for the "inactive" link, the offset time will also contain a nonzero LSL in order to enable a new DB-LSP to be established in advance. Furthermore, the "p-type" of each inservice wavelength channel should be flooded so as to enable OBS edge nodes to perform appropriate wavelength assignments for aggregated bursts according to their QoS

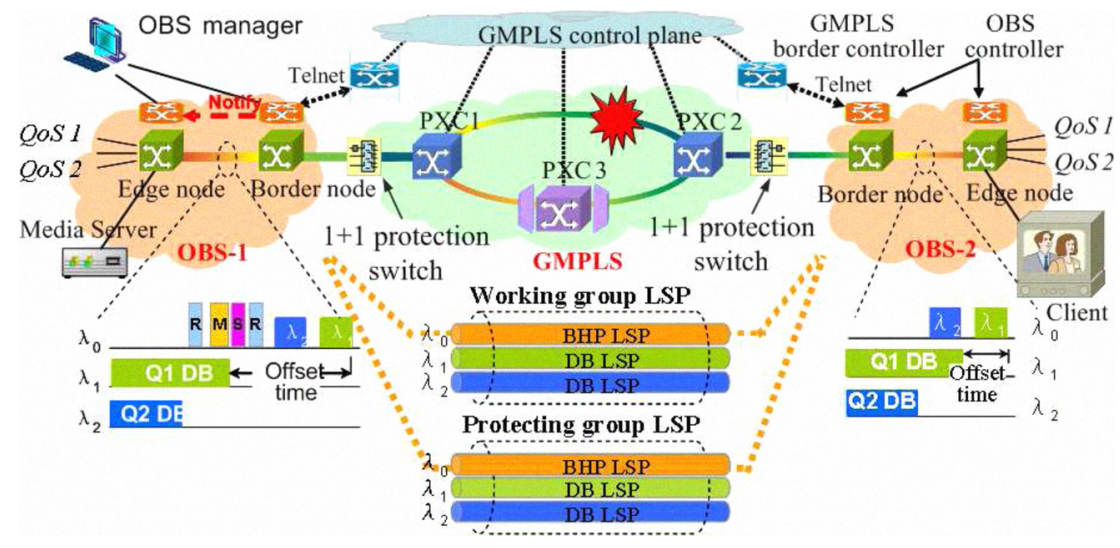

(a)

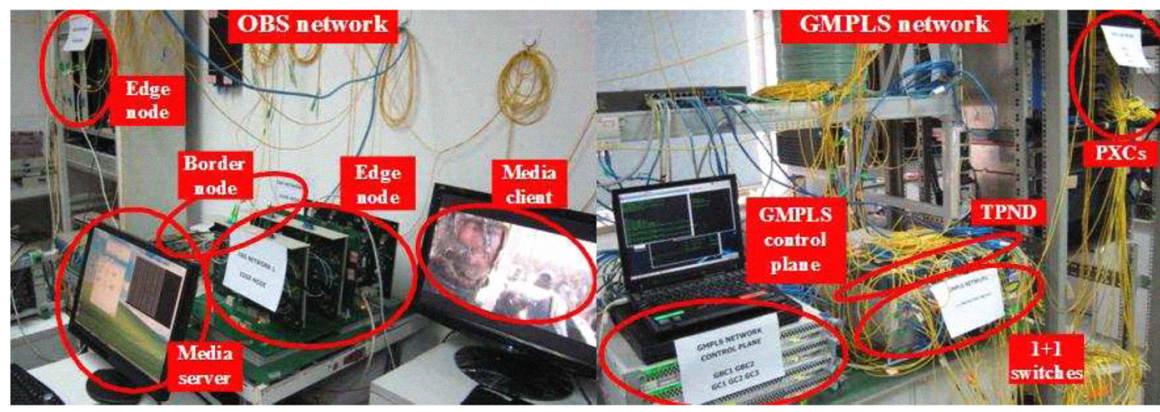

(b)

Figure 3 Multilayer OBS/GMPLS network testbed: (a) network configuration, (b) field picture of demonstration. 
requirements.

Once a network failure is detected, the message as defined in Fig.2 (b) is advertised to indicate the link status modification. Upon receiving such a message, OBS edge node can temporarily stop the traffic during the period of link failure, and also change the offset time from the reference value of working path to that of protecting path when the link is recovered after the protection/restoration operation.

The multilayer OBS/GMPLS network testbed is shown in Fig.3. It consists of one GMPLS-controlled transparent WSON and two labeled OBS client networks. The WSON is constructed by using three photonic cross-connects (PXCs), in which both PXC1 and PXC2 were equipped with an external 1+1 protection switch. Additionally, two GBCs are introduced to interwork with OBS clients in order to provide an ondemand transparent lightpath. Each OBS client network consists of one edge node and one core node. The core node is also enhanced with functionalities to interwork with GMPLS network [6]. The OBS client network is connected to the GMPLS network via the GBC. In addition, a pair of streaming media client and server is attached to the OBS network via $\mathrm{GbE}$ interfaces. In this testbed, for simplicity, only three wavelength $1558.17 \mathrm{~nm}\left(\lambda_{0}\right), 1556.55 \mathrm{~nm}\left(\lambda_{1}\right)$ and $1554.94 \mathrm{~nm}$ $\left(\lambda_{2}\right)$ are assumed available in WSON corresponding to one BHP and two DB wavelengths in OBS networks.

\section{DYNAMIC PROTECTION/RESTORATION AND MULMULTIMEDIA STREAMS TRANSMISSION IN THE MULTILAYER NETWORK}

Due to the bufferless nature, OBS network based on a single way reservation mechanism usually features with a relatively high burst loss rate even at a low traffic load. In order to avoid the unnecessary burst loss and enable OBS networks to offer transparent services applicable to real applications, a lot of contention resolution schemes, such as burst cloning [7] preplanned restoration (like detour mechanism) [8], as well as burst retransmission (BR), were proposed. Similarly, when there is a link or node failure, these schemes may also be applied to recover the affected bursts.

The WSON is responsible for provisioning a dynamic group LSP as the inter-domain link of OBS clients. This group LSP consists of one LSP dedicate to BHPs and multiple LSPs for DB transmission. All LSPs in group LSP could be protected by using conventional GMPLS protection/restoration schemes as standardized by IETF, including $1+1$ protection, preplanned rerouting, and full rerouting [9]. These schemes provide a differentiated value of traffic recovery latency, which typically varies from several tens of milliseconds to several seconds. Considering that various burst streams may have completely different requirements on lightpath survivability, it is desirable for OBS to schedule them onto different wavelength channels (corresponding to DB LSPs in the WSON layer) and then request appropriate protection schemes to the GMPLS network. For example, the burst stream with a high QoS requirement may demand a $1+1$ protection, while the burst stream with a relatively low QoS requirement only demands a preplanned protection. Since BHPs and DBs are tightly coupled and consequently both BHP and DB LSPs need to be recovered simultaneously in case of a failure, it is required to accordingly change the protection type of BHP LSP once a DB LSP with a higher QoS protection type is established upon request.In order to improve the survivability of end-to-end transparent optical connectivity in the multilayer network, some protection/restoration schemes of single OBS and GMPLS layers are combined together, and four classes of protection scheme: OBS retransmit \& GMPLS 1+1, OBS none \& GMPLS $1+1$, OBS retransmit \& GMPLS preplan and OBS none \& GMPLS preplan are implemented. These schemes are supposed to offer differentiated survivability in terms of traffic recovery latency and data loss during the recovery period.

A wide variety of applications may demands for completely different requirements on network survivability. It is desirable to not only enable OBS edge nodes to perform the burst aggregation and burst schedule with considerations of categories of applications, but also to incorporate OBS and GMPLS layers to dynamically apply aforementioned four classes of survivability schemes to each category of traffic.

\section{A. Burst Retransmission in the LOBS Layer}

To evaluate the burst retransmission, a simple OBS network with only two edge nodes (source edge node E1 and destination edge node E2) and one core node (C1) is setup. The group LSP in the GMPLS network can be configured dynamically or statically. A static group LSP is employed to study the delay caused by retransmission, as shown in Fig. 4. Each edge node is connected with $\mathrm{Cl}$ through a pair of fiber links.

The experimental results for burst retransmission are shown in Fig. 5. In the experiment, the length of assembled bursts is set to 625 Kbytes and the offset time is set to $1 \mathrm{~ms}$. It is assumed that the link fault time (TLF) and message propagation delay (TB) are about $120 \mathrm{~ms}$ and $110 \mathrm{~ms}$. The minimum buffer requirement is $230 \mathrm{~ms}$, which amounts to $36 \mathrm{Mbytes}$ for $1.25 \mathrm{Gbps}$ link. In our experiment, more than 200Mbytes SRAM buffer is used. Upon receiving the link recovery message (LRM), both waiting bursts, that are buffered and never being transmitted and requesting bursts, that fail to be transmitted from E1 to E2 due to the link fault are firstly scheduled, and then the newly coming bursts are handled. Thanks to the parallel processing of requesting bursts and waiting bursts, BHPs of a waiting burst and a requesting burst could be sent out in a very short time which makes them seem

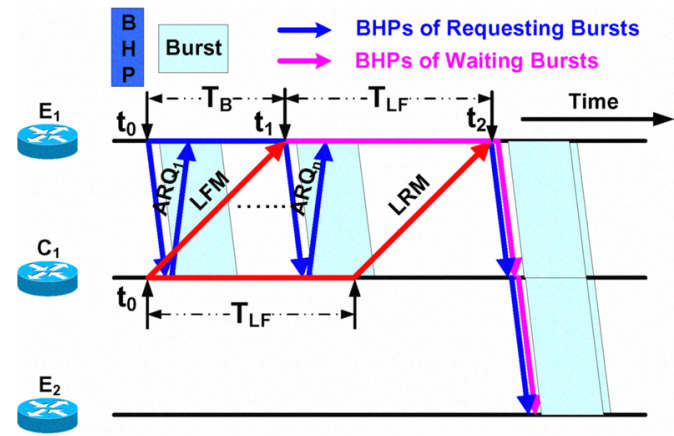

Figure 4 Burst retransmission scheme in the OBS network. 


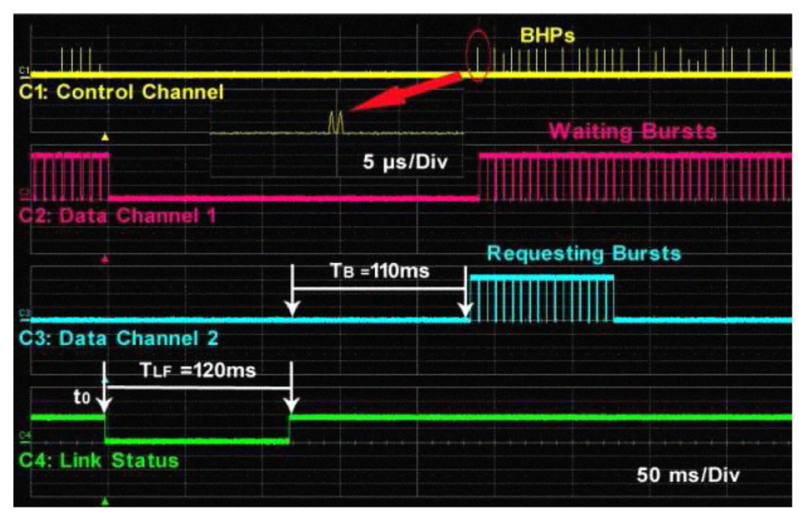

Figure 5. Received BHP/DB pairs in the burst retransmission experiment

to be only one BHP in the large time scale $(50 \mathrm{~ms} / \mathrm{Div})$. But with a small time scale of $5 \mu \mathrm{s} / \mathrm{Div}$ they could be clearly distinguished. The waiting bursts are transmitted in the data channel $\lambda_{1}$ (denoted by $\mathrm{C} 2$ ) and the requesting bursts are retransmitted in the data channel $\lambda_{2}$ (denoted by $\mathrm{C} 3$ ).

\section{B. Dynamic Protection of Group LSP in the GMPLS Layer}

In the experiment, the optical switch used in GMPLS is based on 3D MEMS, whose rising time is less than 10ms. In OBS, a high voltage Electro-optical switch with 30ns rising time is used. A group LSP containing only one BHP LSP without any protection at the channel $\lambda_{0}$ is firstly established along the shortest path PXC1-PXC2. This BHP LSP could be utilized to exchange routing information between the connected OBS client networks. After that, a burst stream with low QoS requirement is injected into the data channel $\lambda_{1}$ and accordingly a request for DB LSP with preplan protection was sent from OBS to GMPLS networks. Receiving this request, the ingress GBC attempted to add a working DB LSP along the path of existing group LSP and also to calculate a diverse path PXC1PXC3-PXC2 containing available wavelengths $\lambda_{0}$ and $\lambda_{1}$ in order to establish preplanned protecting BHP and DB LSPs respectively. It is confirmed that the protection type of $\mathrm{BHP}$ LSP is successfully modified from full rerouting to preplanned, and both BHP and DB traffic are switched from the working to protecting LSPs in about $160 \mathrm{~ms}$ once an intentional fiber cut is introduced in the working link, as shown in Fig. 6. The traffic is also automatically switched back after about $160 \mathrm{~ms}$ once the
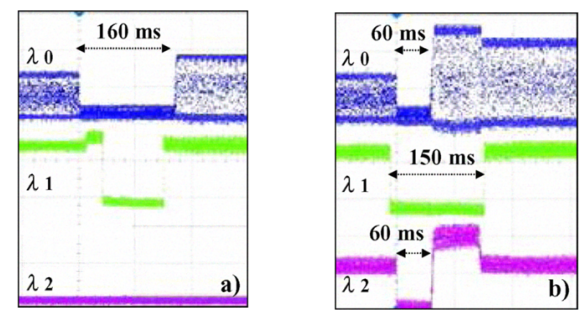

Figure 6. Protection switching of the group LSP consisting of a) one DB LSP with preplanned protection and $b$ ) one additional DB LSP with $1+1$ protection. working link is recovered. The switching time is determined by the signaling delay and the response time of the switch in GMPLS.

\section{Streaming Media Transmission in the Multilayer Network}

In case of a failure, the VoIP service is more sensitive to the network latency and jitter and the high definition video on demand (HD VOD) service is less sensitive to the latency and jitter (when the buffer of player is deep enough), while the data loss may cause a significant quality of user experience (QoE) degradation.

Firstly the impact of different survivability schemes on the streaming media service in case of a link failure is evaluated. For simplicity, the OBS assembly parameters (i.e. time threshold and burst length threshold) are set to be constant. Indeed, the OBS assembly process has a great impact on the streaming media packet in several aspects, e.g., the delay and jitter will be increased if a huge number of media packets were assembled into one large burst. In the experiment, series of streaming media with different bit rates are transmitted using RTP/UDP protocols over the OBS/GMPLS network, and the bit rate varied from hundreds of Kbps to hundreds of Mbps. All of streams last for thirty minutes, during which one time of intentional fiber cut is randomly introduced into the GMPLS working group LSP and four classes of survivability schemes are applied respectively. The delay factor (DF) [10] is introduced here to assess the depth of demanded buffer required for playing streaming media at the client. Fig. 7 (a) and (b) illustrate the DF and packet loss value under different stream bit rates when the four classes of survivability schemes applied. It can be seen that schemes I and III perform worse than schemes II and IV in term of DF, but better than them in term of numbers of IP packet loss. Note that the data loss of scheme III increases to an abnormally large value when the stream bit rate is $240 \mathrm{Mbps}$, due to the fact that the OBS retransmission buffer is not deep enough and an overflow occurs during the recovery period of GMPLS preplanned restoration.

For several mature streaming media services, the DF and data loss are suggested to be certain values. For example, the maximum acceptable data loss for SDTV and VOD services are recommended to be up to five consecutive IP packets per thirty minutes, and the DF introduced within the network is recommended to remain below $50 \mathrm{~ms}$ [11]. Considering this recommendation, it can be concluded that schemes I and III may be more suitable to standard VOD services (namely QoS level 3) when the buffer of the media player is sufficient. Similarly, the priorities of four survivability schemes for other QoS levels of applications, as summarized in Fig. 7 (c), can be obtained.

Secondly, the dynamic protection of multiple QoS level of services is confirmed. When the media stream with QoS level 3 (Q2 in Fig. 3(a)) is injected from the media server, the OBS edge node starts to schedule the data bursts onto channel $\lambda_{2}$. In response, the scheme III (the first priorized one for QoS level 3) is chosen and a working group LSP is established along the shortest path PXC1-PXC2 on channel $\lambda_{0}(\mathrm{BHP})$ and $\lambda_{2}(\mathrm{DB})$, and the corresponding preplanned protecting group LSP along 


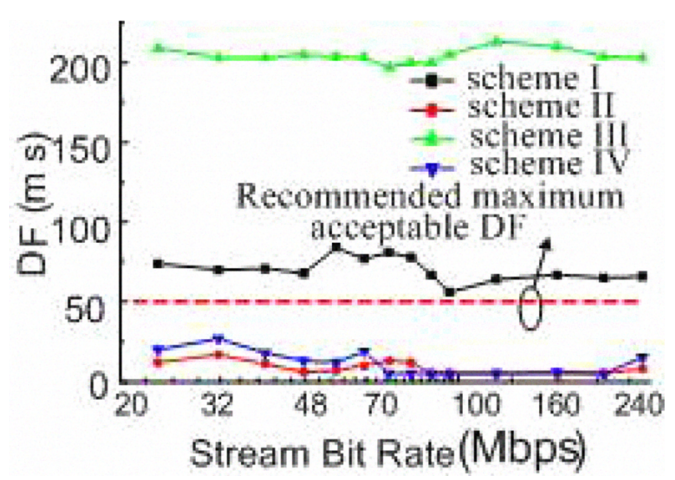

(a)

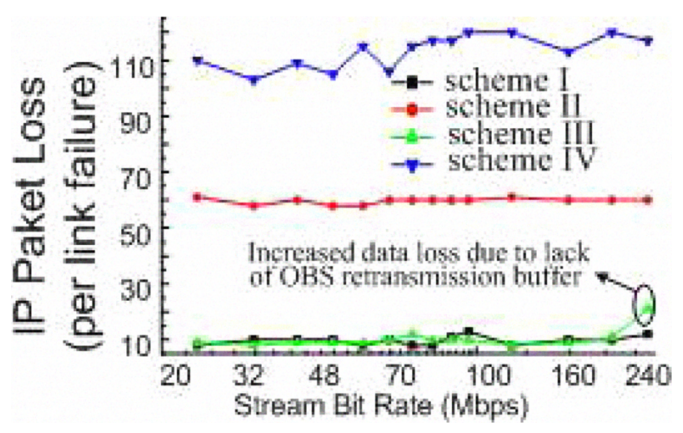

(b)

\begin{tabular}{|c|c|}
\hline QoS Leve & Survikabillity Priority \\
\hline 1 & I $>$ II $>1$ II $>$ IV \\
\hline 2 & II $>$ IV $>$ I $>$ III \\
\hline 3 & III $>1>$ II $>$ IV \\
\hline 4 & $1=$ III $>11>1 V$ \\
\hline
\end{tabular}

(c)

Figure 7. Performances of four type of survivability schemes: (a) delay factor, (b) packet loss per link failure when different survivability schemes applied, (c) prioritized survivability schemes.

a diverse path PXC1-PXC3-PXC2 is also reserved. When another media stream with QoS level 1 is injected, the assembled bursts are scheduled to channel $\lambda_{1}$, and another DB LSP with its corresponding $1+1$ protecting LSP is added into the same group LSP without affecting the previous service. More specifically, the protection type of the BHP LSP is also updated from "preplanned rerouting" to " $1+1$ protection" since it should inherit the survivability schemes of the highest QoS level in the same group LSP. Then when an intentional link failure is introduced in the working link, it is confirmed that the DB traffic on $\lambda_{1}$ and $\lambda_{2}$ are switched from the working LSPs to protecting LSPs in about $60 \mathrm{~ms}$ for " $1+1$ protection" and 160 ms for "preplanned rerouting", respectively, and the lost bursts during this period are retransmitted.

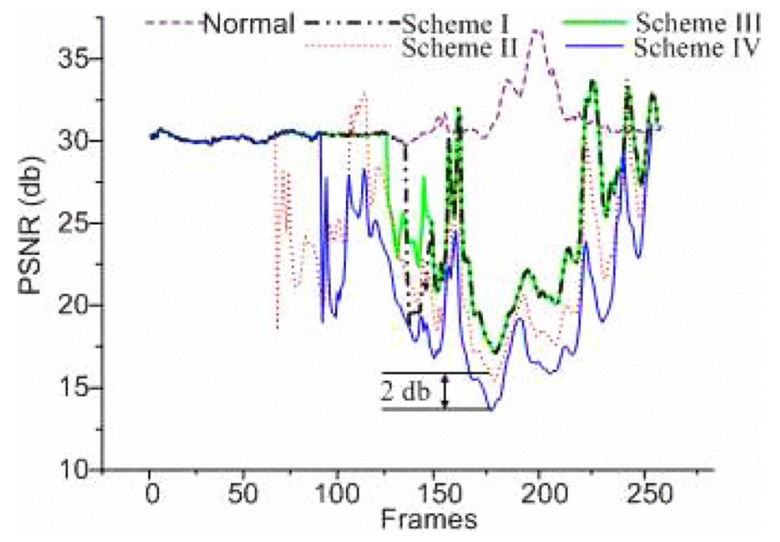

Figure 8. PSNR of received video test sequence.

Finally, a demonstration of a high definition VOD service is carried out on the testbed. The testing video sequence, using a MPEG2 (variable bit rate) codec, has a resolution of $1920 * 1080$, and the average bit rate is $115 \mathrm{Mbps}$ with a peak rate of $217 \mathrm{Mbps}$. During the delivery of the streaming media at the server using RTP/UDP protocol through the OBS/GMPLS testbed, the fluent, clear video is observed at the client without visible error in case of a link failure.

In order to further evaluate the quality of transmitted video in case of a link failure, a background traffic of $200 \mathrm{Mbps}$ as well as a "Foreman" QCIF (Quarter Common Intermediate Format) standard video testing sequence are injected into the testbed, the conventional PSNR (peak signal-noise ratio, equivalent to the visual quality of video frames) is calculated for the received QCIF video. As shown in Fig. 8, the PSNR decreases when the frames are affected in case of a link failure (i.e., some data was lost). Since the buffer of the streaming media player is set deep enough according to the DF, the scheme I and III lose almost the same amount of data, resulting in the same PSNR reduction. For the same reason, the scheme IV achieves a PSNR value about $2 \mathrm{~dB}$ lower than scheme II since more data bursts are lost. The image quality varies with different survivability schemes while link failure occurs. Scheme I has significantly better quality compared with other schemes.

\section{CONCLUSIONS}

This paper investigates the QoS-classified survivability schemes for the end-to-end transparent optical connectivity in the overlay-based OBS/GMPLS multilayer network. Corresponding experimental evaluation is also conducted by using streaming media transmission over a transparent OBS/GMPLS testbed, and a reliable delivery of HD video streaming is successfully demonstrated. Experimental results validate the applicability of such end-to-end transparent optical connectivity to maintaining high quality services by applying the appropriate survivability scheme. 


\section{ACKNOWLEDGMENT}

This work is partly supported by MOST program with grant No.2006DFA11040, 2007AA01Z248, 111 Project (B07005) and PCSIRT with grant No.IRT0609, MOE, China.

\section{REFERENCES}

[1] R. Nejabati, et al., Grid Optical Burst Switched Networks (GOBS), Open Grid Forum, Draft GFD.128.

[2] T. Otani, H. Guo, K. Miyazaki, and D. Caviglia, "Generalized labels of lambda-switching capable label switching routers (LSR)", IETF draft (work in progress), draft-ietf-ccamp-gmpls-g-694-lambda-labels-04.txt, March 2009.

[3] H. Guo, et al, "A testbed for optical burst switching network", in Proc.OFC, OFA6, Mar 2005

[4] H. Guo, et al, "Experimental Demonstration of Interworking GMPLS with OBS Networks" ECOC2007 1.6.3

[5] Y. Yin, et al., "Dynamic protection and restoration supporting QoS in OBS/GMPLS interworking network," in Tech. Digest of European Conference on Optical Communication (ECOC), Brussels, Belgium, Sep. 2008, paper Tu.1.C.6.

[6] P. Huang, H. Guo, W. Zhang, T. Tsuritani, J. Wu and T. Otani, "Experimental implementation of a protocol interface between GMPLS and LOBS testbed," presented at Asia-Pacific Optical Communications Conference (APOC), Wuhan, China, Nov. 2007, paper 6784-16.

[7] X. Huang, et al., "Burst cloning: a proactive scheme to reduce data loss in optical burst-switched networks," in Proc. IEEE ICC2005, May 2005, vol. 3, pp. 1673-1677.

[8] A. Bononi, G.A. Castanon, and O.K. Tonguz, "Analysis of hot-potato networks with wavelength conversion," IEEE/OSA Journal of Lightwave Technology, vol. 17, no. 4, pp. 525-534, Apr. 1999.

[9] J.P. Lang, Y. Rekhter, and D. Papadimitriou, "RSVP-TE extensions in support of end-to-end generalized multi-protocol label switching (GMPLS) recovery," IETF RFC4872, May. 2007.

[10] J. Welch and J. Clark, "A proposed media delivery index (MDI)," IETF RFC4445, Apr. 2006.

[11] T. Rahrer, et al., "Triple-play service quality of experience $(\mathrm{QoE})$ requirements," Technical report (TR-126) of DSL Forum, Dec. 2006. 